\title{
Life-threatening rupture of an external iliac artery pseudoaneurysm caused by necrotizing fasciitis following laparoscopic radical cystectomy: a case report
}

\author{
Shinro Hata*, Ryuta Satoh, Toshitaka Shin, Kenichi Mori, Yasuhiro Sumino, Fuminori Satoh and Hiromitsu Mimata
}

\begin{abstract}
Background: Pseudoaneurysms are caused by trauma, tumors, infections, vasculitis, atherosclerosis and iatrogenic complications. In this paper, we report about a patient with rupture of an external iliac artery pseudoaneurysm, which lead to hemorrhagic shock, after undergoing laparoscopic radical cystectomy and extended pelvic lymphadenectomy.

Case presentation: The patient was a 68-year-old Japanese male diagnosed with invasive bladder cancer. Laparoscopic radical cystectomy and extended pelvic lymphadenectomy were performed. On postoperative day 12 , he developed a high fever and an acute inflammatory response with redness and swelling in the right inguinal region. He was diagnosed with necrotizing fasciitis and underwent debridement. On postoperative day 42, a sudden hemorrhage developed from the open wound in the right inguinal region. He was diagnosed with external iliac artery pseudoaneurysm rupture by computed tomography.
\end{abstract}

Conclusion: These complications occur extremely rarely after cystectomy with pelvic lymphadenectomy. There are no reports to date on these complications following laparoscopic cystectomy with pelvic lymphadenectomy.

Keywords: Bladder cancer, Pseudoaneurysm, Laparoscopic, Pelvic lymphadenectomy

\section{Background}

It is extremely rare for formation and rupture of an iliac artery pseudoaneurysm after pelvic surgery. Usually, pseudoaneurysms are caused by trauma, tumors, infections, vasculitis, atherosclerosis and iatrogenic complications [1]. In this paper, we report about a patient with rupture of an external iliac artery pseudoaneurysm, which lead to hemorrhagic shock, after undergoing laparoscopic radical cystectomy, extended pelvic lymphadenectomy and ileal conduit diversion.

\section{Case presentation}

The patient was a 68-year-old Japanese male diagnosed with invasive bladder cancer with clinical stage T3a N0 M0 (Figure 1). His body mass index (BMI) was $17.4 \mathrm{~kg} / \mathrm{m}^{2}$

\footnotetext{
* Correspondence: hatashinn@oita-u.ac.jp

Department of Urology, Faculty of medicine, Oita University, Idaigaoka 1-1, Hasama-cho, Yufu, Oita Prefecture 879-5593, Japan
}

and he had a history of alcoholic hepatitis. Liver function before the surgery was good. Laparoscopic radical cystectomy, extended pelvic lymphadenectomy and ileal conduit diversion were performed. No intraoperative complications were observed, the surgery time was 679 minutes, and the estimated blood loss was $340 \mathrm{ml}$. Pathological stage was pT3a pNO. Although the early postoperative course was favorable, the patient developed a high fever and an acute inflammatory response with redness and swelling in the right inguinal region on postoperative day (POD) 12 (Figure 2). Intravenous antibiotics were initiated but skin necrosis ensued. The patient was diagnosed with necrotizing fasciitis and retroperitoneal abscess by computed tomography $(\mathrm{CT})$ (Figure 3). Then the patient underwent debridement on POD 13 (Figure 4). At this time the external iliac artery could not be identified. After debridement, even though 


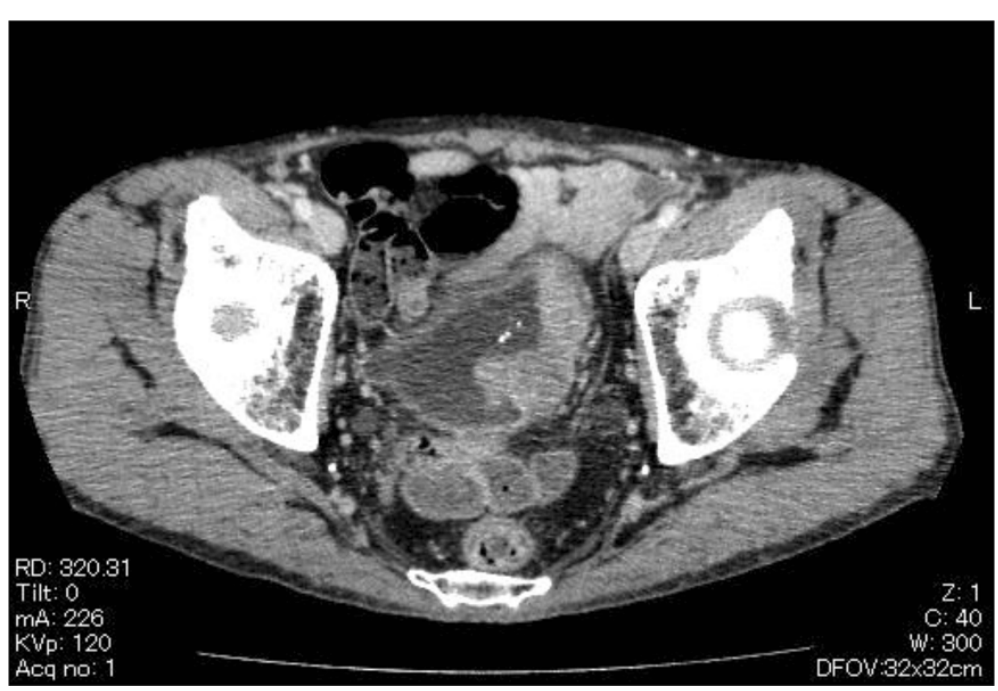

Figure 1 Preoperative CT findings of the pelvis; CT showed the invasive bladder cancer (clinical stage T3a) and the normal external iliac artery.

the local inflammatory findings were improved, the levels of C-reactive protein (CRP) remained elevated $(3-4 \mathrm{mg} / \mathrm{dl})$. Six weeks after initial surgery, there was noted to be excessive spontaneous bleeding from the right inguinal wound, resulting in hemorrhagic shock. The patient was diagnosed with external iliac artery pseudoaneurysm rupture by CT (Figure 5). Bypass graft surgery was performed using the great saphenous vein (Figure 6). Methicillin-resistant Staphylococcus aureus (MRSA) was cultured from the wound, and the patient's condition slowly improved on intravenous daptomycin. A pedunculated femoral flap and split-thickness skin graft were performed to cover the inguinal wound, and the patient was discharged home 12 weeks after initial

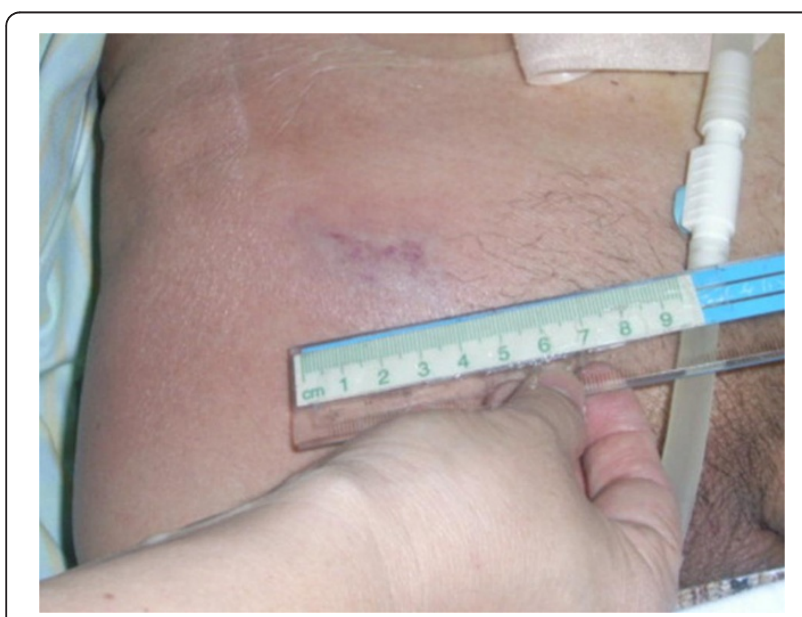

Figure 2 Appearance of right inguinal region; redness and swelling were recognized. surgery. At twelve months follow up, there was no evidence of aneurysm recurrence and no prolonged limb deficit.

\section{Discussion}

Pseudoaneurysms are a result of damage to the vascular wall due to factors such as trauma, tumor, infection, vasculitis, atherosclerosis, or iatrogenic injury [1]. The iliac artery is a rare site of the onset, accounting for 3 to $18 \%$ of all cases. Pelvic surgery rarely results in formation or rupture of iliac artery pseudoaneurysms, and the frequency is unknown [2,3]. Simon et al. have only reported one case of pseudoaneurysm formation in the common iliac artery associated with open radical cystectomy [4]. Vascular injury is rare in laparoscopic cystectomy, with a rate of 0 to $3.7 \%$ [5-7].

Rapid revascularization is usually required since pseudoaneurysm rupture causes shock due to heavy bleeding. Endovascular interventions include surgical revascularization (vascular grafts and autologous vessels), and more

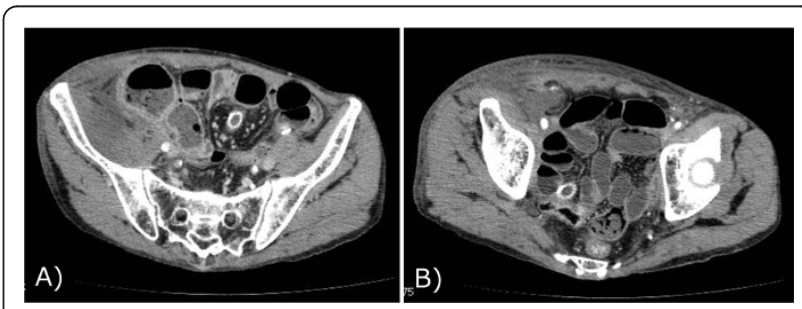

Figure 3 CT findings before the debridement; A) showed the retroperitoneal abscess. B) showed the normal external iliac artery. 


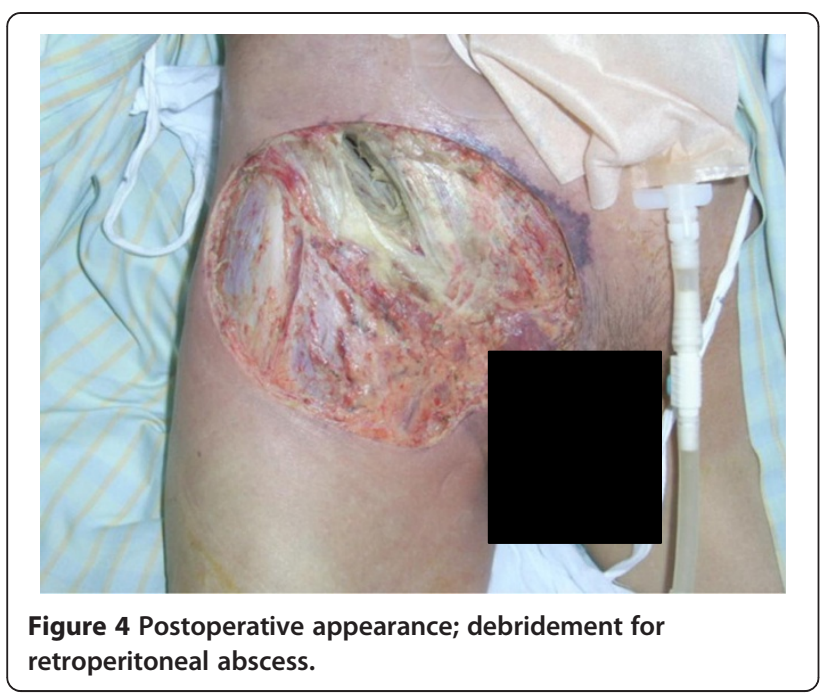

recently, stent grafts. Ricciardi et al. reported a case of external iliac artery pseudoaneurysm rupture following pelvic lymphadenectomy for cervical cancer [2]. They performed endovascular intervention using a covered stent, and advocate their use as first-line treatment in these kinds of cases, as it is possible to achieve faster control of bleeding than with surgical techniques.

The present case was an infectious pseudoaneurysm that spread from a retroperitoneal abscess, and thus foreign objects should not be left within the body. At the time of debridement, Staphylococcus epidermides was detected in a subcutaneous pus swab and MRSA was detected from a retroperitoneal pus swab. Therefore, we

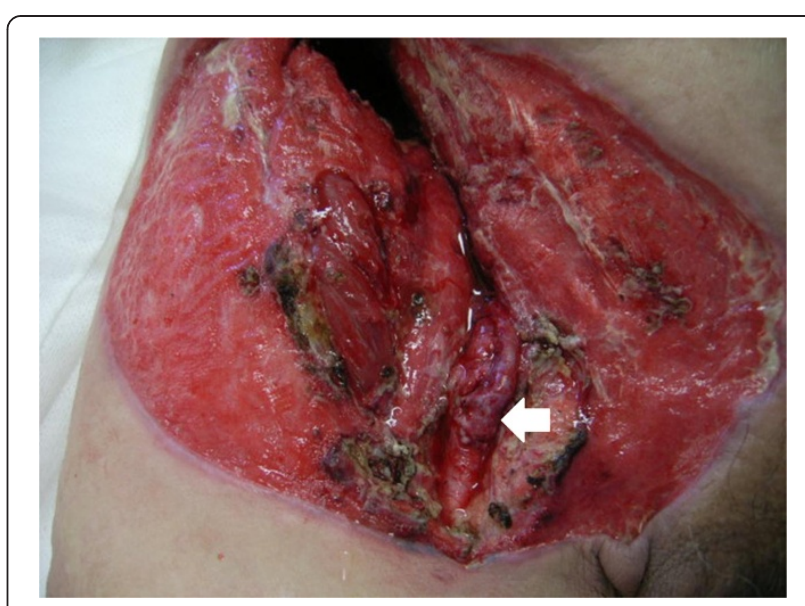

Figure 6 Postoperative appearance (bypass surgery); the arrow showed the great saphenous vein. conducted revascularization using an autologous graft from the great saphenous vein without a covered stent or vascular graft.

In this case, the factors leading to pseudoaneurysm rupture were thought to be the spread of infection from the retroperitoneal abscess and the weakening of the vascular wall associated with the lymphadenectomy. The factors that triggered the formation of the retroperitoneal abscess are unclear. We suggest that the cause of pseudoaneurysm formation was microscopic injury of the external iliac artery which was not detected during surgery. Furthermore, the vascular wall became brittle due to $M R S A$ infection, ultimately leading to rupture.

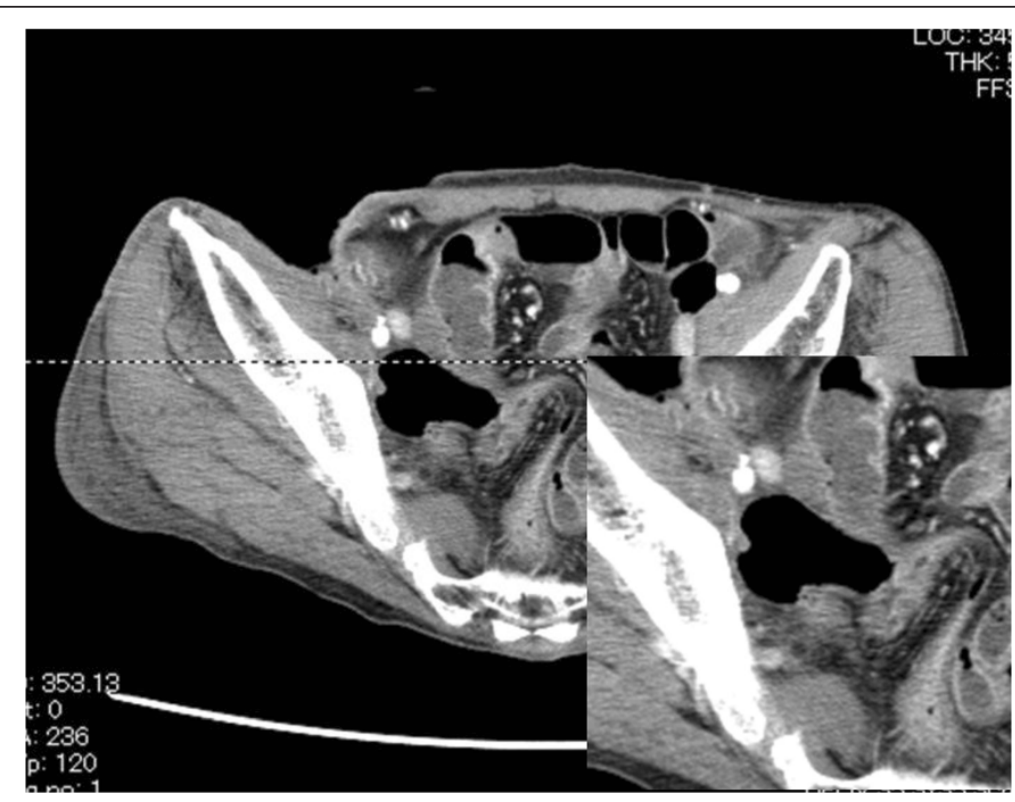

Figure $5 \mathrm{CT}$ findings of the pelvis; CT showed the pseudoaneurysm of external iliac artery. 


\section{Conclusions}

External iliac artery pseudoaneurysms are rare complications of laparoscopic radical cystectomy with pelvic lymphadenectomy. When recognized and treated promptly, good long-term functional outcomes can be achieved.

\section{Consent}

Written informed consent was obtained from the patient for publication of this case report and any accompanying images. A copy of the written consent is available for review by the Editor-in-Chief of this journal.

\section{Abbreviations \\ POD: Postoperative days; CT: Computed tomography; BMI: Body mass index; MRSA: Methicillin-resistant Staphylococcus aureus.}

\section{Competing interests}

The authors declare that they have no competing interests.

\section{Authors' contributions}

SH performed the majority of this study and drafted the manuscript. RS and TS surveyed the literature. KM and HM critically revised the manuscript. YS,

FS, and HM participated in the design and interpretation of this study under supervision. All authors read and approved the final manuscript.

\section{Acknowledgements}

We are grateful to Hiromitsu Mimata for his dedicated work in the patient management.

Received: 18 December 2013 Accepted: 7 May 2014

Published: 10 May 2014

\section{References}

1. Sueyoshi E, Sakamoto I, Nakashima K, Minami K, Hayashi K: Visceral and peripheral arterial pseudoaneurysms. AJR Am J Roentgenol 2005, 185(3):741-749.

2. Enzo R, Giampaolo Di M, Paolo M, Mauro S, Antonio F, Marina J, Borut K, Massimo M: Life-threatening bleeding after pelvic lymphadenectomy for cervical cancer: endovascular management of ruptured false aneurysm of the external iliac artery. World J Surg Oncol 2012, 10:149.

3. Huang W-Y, Huang C-Y, Chen C-A, Hsieh C-Y, Cheng W-F: Ruptured pseudoaneurysm of the external iliac artery in an advanced cervical cancer patient treated by endovascular covered stent placement. J Formos Med Assoc 2008, 107(4):348-351.

4. Hampson SJ, Buckenham T, Patel A, Woodhouse CR: False aneurysm of the common iliac artery after pelvic lymphadenectomy: management of an unusual complication. J Urol 1996, 154:201-202.

5. Huang J, Lin T, Liu H, Kewei X, Zhang C, Jiang C, Huang H, Yao Y, Guo Z, Xie W: Laparoscopic radical cystectomy with orthotopic ileal neobladder for bladder cancer: oncologic results of 171 cases with a median 3-year follow-up. Eur Urol 2010, 58:442-449.

6. Desai MM, Berger AK, Brandina RR, Zehnder P, Simmons M, Aron M, Skinner EC, Gill IS: Robotic and laparoscopic high extended pelvic lymph node dissection during radical cystectomy: technique and outcomes. Eur Urol 2012, 61:350-355.

7. Christopher S, Nasreldin M, Stefano A, Gerit T, Vincenzo Maria A, Paolo F, Francesco G: Laparoscopic radical cystectomy with extracorporeal ileal neobladder for muscle-invasive urothelial carcinoma of the bladder: technique and short-term outcomes. World J Urol 2014, 32(2):407-412.

\section{doi:10.1186/1756-0500-7-290}

Cite this article as: Hata et al.: Life-threatening rupture of an external iliac artery pseudoaneurysm caused by necrotizing fasciitis following laparoscopic radical cystectomy: a case report. BMC Research Notes 2014 7:290.

\section{Submit your next manuscript to BioMed Central and take full advantage of:}

- Convenient online submission

- Thorough peer review

- No space constraints or color figure charges

- Immediate publication on acceptance

- Inclusion in PubMed, CAS, Scopus and Google Scholar

- Research which is freely available for redistribution 